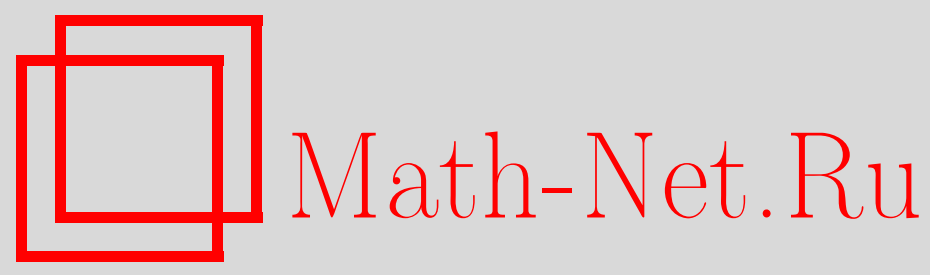

Р. С. Исмагилов, П. С. Саркисян, О квазиоднородных струнах, Матем. заметки, 2005, том 78, выпуск 2, 317-320

DOI: https://doi.org/10.4213/mzm2590

Использование Общероссийского математического портала Math-Net.Ru подразумевает, что вы прочитали и согласны с пользовательским соглашением http://www. mathnet.ru/rus/agreement

Параметры загрузки:

IP: 18.207 .199 .55

26 апреля 2023 г., 09:58:51

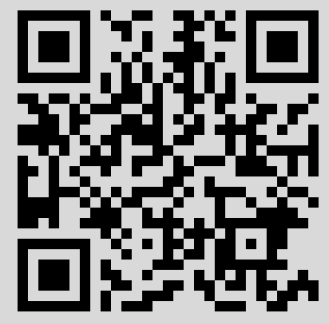




\section{О КВАЗИОДНОРОДНЫХ СТРУНАХ}

\section{Р. С. Исмагилов, П. С. Саркисян}

1. Постановка задачи; формулировка результата. Колебания струны с закрепленными концами описываются спектральной задачей

$$
-y^{\prime \prime}(x)=\lambda^{2} \rho(x) y(x), \quad y(0)=y(b)=0, \quad 0 \leqslant x \leqslant b ;
$$

здесь функция $\rho(x), 0 \leqslant x \leqslant b$ (плотность струны), предполагается положительной и кусочно-непрерьвной. Согласно теории обратной задачи, функция $\rho(x)$ не определяется однозначно по спектру задачи (1). Цель этой простой заметки прежде всего методическая. Мы обращаем внимание на то, что указанное обстоятельство имеет место уже для кусочно-постоянных плотностей. С целью оттенить это обстоятельство мы выделяем класс струн с кусочно-постоянной плотностью, для которых спектр колебаний совпадает со спектром однородной струны. Условия, определяющие такой класс, записываются в виде простых алгебраических соотношений; вывод этих соотношений и будет нашей целью.

Как будет показано в следующей работе одного из авторов, несложные алгебраические манипуляции над многочленами позволяют решить и более сложные задачи о струнах с указанным свойством, скажем, задачи о восстановлении плотности такой струны, если известна плотность ее части. Предлагаемая заметка содержит необходимую подготовку для обращения к этим задачам.

В связи с задачей восстановления необходимо упомянуть о задаче, рассмотренной Крейном [1]. В его работе рассматривается восстановление плотности при условии, что эта плотность симметрична относительно середины струны; это позволяет восстановить плотность струны однозначно.

Итак, мы рассматриваем функцию $\rho(x)$, при $x \in[0, b]$ определяемую условиями

$$
\rho(x)=a_{k}^{2} \text { при } x \in\left[c_{k-1}, c_{k}\right], \quad k=1, \ldots, n,
$$

где

$$
0=c_{0}<c_{1}<\cdots<c_{n}=b, \quad a_{k}>0, \quad k=1, \ldots, n .
$$

Положим $l_{k}=c_{k}-c_{k-1}$. Исследование задачи (1) существенно упрощается, если наложить следуюшие ограничения:

$$
a_{k} l_{k}=\alpha, \quad k=1, \ldots, n,
$$

для некоторого фиксированного $\alpha$. В дальнейшем это условие считается выполненным. Заметим, что если условие (2) нарушено, но величины $a_{k} l_{k}$ становятся рациональньпи при умножении на фиксированное число, то условие (2) достигается путем подразбиения каждого отрезка $\left[c_{k-1}, c_{k}\right]$ на части одинаковой длины.

Вся информация о такой струне может быть записана строкой параметров $a_{1}, \ldots, a_{n}, \alpha$.

Однородная струна, как частный случай струны с кусочно-постоянной плотностью, может быть определена строкой $a, \alpha$, где $a>0, \alpha>0$.

ОПРЕДЕЛЕНИЕ. Назовем струну квазиоднородной, если ее спектр совпадает со спектром однородной струны.

Основной результат заметки заключается в следующей теореме.

ТеОРема. Струна $a_{1}, \ldots, a_{n}, \alpha$ квазиоднородна тогда и только тогда, когда для всех $m$ таких, что $1 \leqslant m \leqslant n, m$ нечетно, величины

$$
\frac{1}{C_{n}^{m}} \cdot \sum_{i_{1}<\cdots<i_{m}} \frac{a_{i_{2}} \cdots a_{i_{m-1}}}{a_{i_{1}} \cdots a_{i_{m}}}
$$


равны одной и той же не зависящей от т константе.

Условимся задавать квазиоднородную струну строкой $\left(a_{1}, \ldots, a_{n}\right)$, опуская параметр $\alpha$; этот параметр не влияет на свойство квазиоднородности.

Запишем условия квазиоднородности для струн с числом звеньев, не превосходящим пяти.

Трехзвенная струна:

$$
\frac{a_{2}}{a_{1} a_{3}}=\frac{1}{3}\left(\frac{1}{a_{1}}+\frac{1}{a_{2}}+\frac{1}{a_{3}}\right) .
$$

Можно взять, например, $(1,3,6)$.

Четырехзвенная струна:

$$
\frac{a_{2}}{a_{1} a_{3}}+\frac{a_{2}}{a_{1} a_{4}}+\frac{a_{3}}{a_{1} a_{4}}+\frac{a_{3}}{a_{2} a_{4}}=\frac{1}{a_{1}}+\frac{1}{a_{2}}+\frac{1}{a_{3}}+\frac{1}{a_{4}} .
$$

Можно взять, например, $(1,2,2,4)$.

Пятизвенная струна:

$$
\frac{a_{2} a_{4}}{a_{1} a_{3} a_{5}}=\frac{1}{C_{5}^{3}} \sum_{i_{1}<i_{2}<i_{3}} \frac{a_{i_{2}}}{a_{i_{1}} a_{i_{3}}}=\frac{1}{5} \sum_{i} \frac{1}{a_{i}} .
$$

Можно взять, например, $(6,3,5,20,12)$.

2. Доказательство теоремы. 2.1. Вывод уравнения спектра закрепленной струны с кусочно-постоянной плотностью. Рассмотрим произвольное решение $y(x, \lambda)$ уравнения (1). Этому решению поставим в соответствие вектор-строку

$$
z(x, \lambda)=\left(i \lambda \cdot y(x, \lambda), y_{x}^{\prime}(x, \lambda)\right)
$$

Для любого отрезка $\left[\beta_{1}, \beta_{2}\right] \subset[0, b]$ определим матрицу перехода $M=M\left(\beta_{1}, \beta_{2} ; \lambda\right)$ следующим условием:

$$
z\left(\beta_{2}, \lambda\right)=z\left(\beta_{1}, \lambda\right) \cdot M\left(\beta_{1}, \beta_{2}, \lambda\right)
$$

для любого решения $y(x, \lambda)$ уравнения $(1)$.

Пусть теперь $\rho(x)$ кусочно-постоянна.

Матрица перехода $M_{k}=M\left(c_{k-1}, c_{k}, \lambda\right)$ примет вид

$$
M_{k}=\frac{1}{2}\left[\begin{array}{cc}
e^{i \lambda \alpha}+e^{-i \lambda \alpha} & a_{k}\left(e^{i \lambda \alpha}-e^{-i \lambda \alpha}\right) \\
a_{k}^{-1}\left(e^{i \lambda \alpha}-e^{-i \lambda \alpha}\right) & e^{i \lambda \alpha}+e^{-i \lambda \alpha}
\end{array}\right]
$$

Пусть $M=\left(M_{i j}\right)_{i, j=1}^{2}$ - матрица перехода для всего отрезка $[0, b]$. Очевидно, что $M=$ $M_{1} \cdots M_{n} ;$ условимся записьвать это произведение в виде $\prod_{k=1}^{n} M_{k}$.

Уравнение спектра для струны с закрепленными концами имеет вид $M_{21}=0$. Выполним преобразования, позволяющие получить явный вид члена $M_{21}$. Для этого прежде всего сделаем замену переменных $x=e^{i \lambda \alpha}$, тогда

$$
M_{k}=\frac{1}{2}\left[\begin{array}{cc}
x+x^{-1} & a_{k}\left(x-x^{-1}\right) \\
a_{k}^{-1}\left(x-x^{-1}\right) & x+x^{-1}
\end{array}\right]=\frac{1}{2}\left(\left(x+x^{-1}\right) E+\left(x-x^{-1}\right) A_{k}\right),
$$

где $E$ - единичная матрица,

$$
A_{k}=\left[\begin{array}{cc}
0 & a_{k} \\
a_{k}^{-1} & 0
\end{array}\right]
$$

$\mathrm{C}$ целью упрощения дальнейших вычислений введем множество $D=\{0,1\}$, а также множество $D^{n}$, состоящее из всех булевых векторов размерности $n$. Для вектора $b=\left(b_{1}, \ldots, b_{n}\right) \in D^{n}$ положим $\|b\|=b_{1}+\cdots+b_{n}$. Введем также множество $D_{m}^{n}=\left\{b \in D^{n}:\|b\|=m\right\}$. 
Теперь можно записать матрицы $M_{k}$ и $M$ в виде

$$
\begin{aligned}
M_{k} & =\frac{1}{2} \sum_{b_{k} \in D}\left(x+\left(1-2 b_{k}\right) x^{-1}\right) A_{k}^{b_{k}}, \\
M & =\frac{1}{2^{n}} \sum_{b \in D^{n}}\left(\prod_{k=1}^{n}\left(x+\left(1-2 b_{k}\right) x^{-1}\right) \cdot \prod_{\substack{n \\
=1}}^{n} A_{k}^{b_{k}}\right) .
\end{aligned}
$$

Если $b \in D_{m}^{n}$, то

$$
\prod_{k=1}^{n}\left(x+\left(1-2 b_{k}\right) x^{-1}\right)=\left(x-x^{-1}\right)^{m}\left(x+x^{-1}\right)^{n-m} .
$$

Положим

$$
G_{m}=\sum_{b \in D_{m}^{n}} \prod_{k=1}^{n} A_{k}^{b_{k}}
$$

Получаем

$$
M=\frac{1}{2^{n}} \sum_{m=0}^{n}\left(x-x^{-1}\right)^{m}\left(x+x^{-1}\right)^{n-m} G_{m} .
$$

Напомним, что наша цель - вычисление элемента $M_{21}$ матрицы $M=\left(M_{i j}\right)_{i, j=1}^{2}$. Рассмотрим матрицу $\prod_{k=1}^{n} A_{k}^{b_{k}}, b \in D_{m}^{n}$, входящую в сумму (3). Нетрудно заметить, что если $m$ четно, то ее левый нижний элемент равен нулю; если же число $m$ нечетно, то этот элемент имеет вид

$$
\prod_{k=1}^{n} a_{k}^{b_{k}^{*}}, \quad \text { где } b_{k}^{*}=b_{k}(-1)^{b_{1}+\cdots+b_{k}}, \quad k=1, \ldots, n
$$

(таким образом, для получения вектора $b^{*}=\left(b_{1}^{*}, \ldots, b_{n}^{*}\right)$ из вектора $b=\left(b_{1}, \ldots, b_{n}\right)$ следует оставить без изменения нулевые координаты вектора $b$, а первую ненулевую координату вектора $b$ заменить на -1 ; далее ненулевые координаты вектора $b^{*}$ принимают попеременно значения $\left.1,-1\right)$.

Теперь из равенства (3) следует, что левый нижний элемент матрицы $G_{m}$ (обозначим его через $F_{m}$ ) выражается формулой

$$
F_{m}=\sum_{\|b\|=m} \prod_{k=1}^{n} a_{k}^{b_{k}^{*}}
$$

Приведенное выше описание вектора $b^{*}=\left(b_{1}^{*}, \ldots, b_{n}^{*}\right)$ позволяет записать последнюю сумму в виде

$$
F_{m}=\sum_{i_{1}<\cdots<i_{m}} \frac{a_{i_{2}} \cdots a_{i_{m-1}}}{a_{i_{1}} \cdots a_{i_{m}}} .
$$

Наконец, из формулы (4) находим исходный элемент $M_{21}$ матрицы $M$; для краткости обозначим его через $\varphi(x)$ :

$$
\varphi(x)=\frac{1}{2^{n}} \sum_{\substack{0 \leqslant m \leqslant n \\ m \text { неч. }}}\left(x-x^{-1}\right)^{m}\left(x+x^{-1}\right)^{n-m} F_{m} .
$$

Уравнение спектра примет вид $\varphi(x)=0, x=e^{i \lambda \alpha}$.

Для однородной струны, заданной параметром $\alpha_{1}$, уравнение спектра

$$
e^{i \lambda \alpha_{1}}-e^{-i \lambda \alpha_{1}}=0
$$


2.2. Условия квазиоднородности. Квазиоднородность струны означает, что функции $\varphi\left(e^{i \lambda \alpha}\right)$ и $e^{i \lambda \alpha_{1}}-e^{-i \lambda \alpha_{1}}$ имеют одни и те же нули для некоторого числа $\alpha_{1}>0$.

Нетрудно видеть, что это равносильно тому, что $\varphi\left(e^{i \lambda \alpha}\right)=C \cdot\left(e^{i \lambda \alpha_{1}}-e^{-i \lambda \alpha_{1}}\right)$ при $\lambda \in \mathbb{C}$ (для некоторого $C \neq 0$ ). Отсюда следует, что $\alpha_{1}=n \alpha$; таким образом, указанные условия представляются в виде $\varphi(x)=C \cdot\left(x^{n}-x^{-n}\right)$.

Итак, условие квазиоднородности выражается равенством

$$
\frac{1}{2^{n}} \sum_{\substack{0 \leqslant m \leqslant n \\ m \text { неч. }}}\left(x-x^{-1}\right)^{m}\left(x+x^{-1}\right)^{n-m} \cdot F_{m}=C \cdot\left(x^{n}-x^{-n}\right) .
$$

Перепишем это равенство в виде

$$
\frac{\left(x+x^{-1}\right)^{n}}{2^{n}} \cdot \sum_{\substack{0 \leqslant m \leqslant n \\ m \text { нeч. }}}\left(\frac{x-x^{-1}}{x+x^{-1}}\right)^{m} \cdot \frac{F_{m}}{C}=x^{n}-x^{-n}
$$

и введем переменную

$$
t=\frac{x-x^{-1}}{x+x^{-1}}=\frac{x^{2}-1}{x^{2}+1},
$$

тогда $x^{2}=(1+t) /(1-t)$, и наше равенство примет следующий вид:

$$
\sum_{\substack{1 \leqslant m \leqslant n \\ m \text { неч. }}} t^{m} \cdot \frac{F_{m}}{C}=(1+t)^{n}-(1-t)^{n} .
$$

Отсюда

$$
F_{m}=\gamma \cdot C_{n}^{m}, \quad m \text { нечетно, } \quad 1 \leqslant m \leqslant n,
$$

где $\gamma=2 C$. Тем самым, теорема доказана.

\section{СПИСОК ЦИТИРОВАННОЙ ЛИТЕРАТУРЫ}

1. Крейн М. Г. // Докл. АН СССР. 1951. Т. 76. № 3. С. 345-348.

Московский государственный технический университет им. Н. Э. Баумана

E-mail: ismagil@serv.bmstu.ru, sarkisyan@vniieto.ru 